\title{
Improvement of PEAR signaling in energy efficient Delay Tolerant Wireless Sensor Network
}

\author{
Quentin Vey ${ }^{1}$, Pascal Berthou ${ }^{12}$, Thierry Gayraud ${ }^{1} 2$ \\ ${ }^{1}$ CNRS, LAAS, 7 avenue du colonel Roche, F-31400 Toulouse, France \\ 2Univ de Toulouse, a INSA, b UPS, c LAAS, F-31400 Toulouse, France \\ \{vey,gayraud,berthou\}@laas.fr
}

\begin{abstract}
Energy consumption is a critical point for sensor networks, which are located in remote places without access to the power grid and must harvest their own energy. The same networks may also have to use Delay Tolerant Networking technologies if they can't have a permanent connectivity. The PEAR routing algorithm was proposed to address the latter problem. In this paper, we propose an optimization of the signaling information diffusion for this particular algorithm, in order to reduce it's energy consumption. This new algorithm matches some specific characteristics that can be found in agricultural monitoring networks. Its performances have been simulated, and it multiplies network's packet efficiency by 2.77 while maintaining the same successful transmission rate as the original PEAR algorithm or Epidemic algorithm. It increase the usefulness of this algorithm in low power networks and low loaded networks.
\end{abstract} Routing

Keywords-PEAR; PEAR OPD; DTN; Sensor Network;

\section{INTRODUCTION}

Nowadays, industrial companies, researcher and public services aim to deploy large scale wireless sensor networks to gather data over wide facilities, experiment fields and area to monitor. These networks are made of small electronic devices (called sensor nodes), able to sense their environment (temperature sensor, moist sensor...) and able to collaboratively transfer this data to a specific node (often a monitoring station).

In some cases, where connectivity is not always available, DTN (Delay/Disruption Tolerant Networking) techniques are used. Originally created for interplanetary communication where the great distance induces large delays, this paradigm can also be used when the movement of the nodes creates disruption in the connectivity. Several routing algorithm have been developed to match several uses of those DTN networks, for example to grant access to the internet to remote villages (DAKNET [1]), to monitor wildlife (Zebranet [2]) or over a sensor network.

In a such DTN, it has been proposed to use the so-called "data MULE" (Mobile Ubiquitous LAN Extension) to enhance the connectivity of some remote areas : they are special mobile nodes, whose role is to travel between unconnected parts of the network, carrying the messages from one node to another if this can't be done directly.
In the case of agricultural monitoring, even if the sensors are sparsely deployed, the size of the area to cover is so huge that the number of node can become very important. This leads to several concerns : the routing algorithm used in these networks must be scalable and the cost of the nodes must be as low as possible. Also, because of the lack of access to communication or power infrastructure, the static nodes will have either to store enough energy in batteries or to harvest energy from its surrounding (e.g. with solar panels) in order to power up its transceiver. One way to reduce the costs of the nodes is to reduce the size of the batteries or the size of the solar panel. To do so, one have to reduce the energy consumption of the node.

In this paper, we investigate the use of a particular routing algorithm for DTN-based wireless sensor network applied to agricultural monitoring, Potential-based Entropy Adaptive Routing (PEAR), and propose an optimized way of disseminating its routing information. The PEAR algorithm was proposed, and tested for agricultural monitoring purposes, and rely on a particular architecture : a lot of nodes are static, deployed in the fields, and some mobile nodes ensure the connectivity by traveling between the different areas of the monitored region (mounted on agricultural vehicles for example). The performance of this algorithm has been proved in [3], an experiment has been conduct in [4], but no papers have been found on the energy consumption due to PEAR signaling.

After presenting the scenario and assumption we made in II, we will shortly describe the routing algorithm, PEAR, in III. Then we will propose an improvement of the potential information diffusion in IV and evaluate his performances in $\mathrm{V}$. These results will be discussed in VI, and our conclusion will be given in VII.

\section{STUDY SCENARIO}

\section{A. Conditions}

In our scenario, we consider a farmer who wants to monitor his fields (e.g. soil moist, nitrogen levels ... ), in order to optimize the use of water and fertilizer. To do so, several sensor nodes are deployed within the fields, and agricultural vehicles are equipped with a node (the data MULE). When it travels along the field, the mobile node communicates with the 
static nodes. When it arrives at the main station, the data are collected for analysis.

\section{B. Assumptions on energy consumption}

In order to estimate the network electrical consumption, we made the following assumptions :

- The electrical consumption of the radio transceiver of a node is one of the main source of a node's energy consumption, whether it is in receive or transmit mode.

- Due to the number of low-power MAC protocol available, we assume that the energy consumption of transceiver is proportional to the number of packets send or to the amount of data send.

- Because the mobile nodes are mounted on a vehicle, we assume that they have access to a sufficient source of power (e.g. if they are connected on the $12 \mathrm{~V} \mathrm{DC}$ socket), so the electric consumption of mobile nodes was excluded from our study. The main station being located in a building, we assume that it's plugged in a wall socket and also exclude it from our count.

\section{Goals}

Our goal is to reduce the amount of energy greedy network resource used by the PEAR protocol in low loaded network, especially by its signaling. To do so, we count the number of packets and the amount of data send to the layer 2 by the network layer. In this count we include both "data" messages, carrying application level data, network signaling and discovery messages, carrying routing information.

\section{ROUTING ALGORITHM DESCRIPTION}

PEAR was proposed in [3] to enable routing in a DTN sensor network, and a real-world experiment was conducted in [4]. It relies on a potential field to achieve its routing. One of its main features is the self-configuration of the network, and its self re-configuration if the MULE trajectory changes. Let $V^{d}(n, t)$ be the potential of node $n$ for destination $d$ at time $t$ and $\operatorname{nbr}(n)$ the set of neighbors of $n$. In this definition, $n \in n b r(n)$. The potential field is recursively build every time step (every $10 \mathrm{~s}$ in our experiment) for every node $n$ according to $(1)$ :

$$
\begin{aligned}
V^{d}(n, t+1) & =V^{d}(n, t)+\rho \\
& +D \times \min _{k \in n b r(n)}\left\{V^{d}(k, t)-V^{d}(n, t)\right.
\end{aligned}
$$

Potentials are initialized at $0\left(V^{d}(n, 0)=0\right)$ and potential of node $n$ for itself is tied to $0\left(V^{n}(n, t)=0\right)$. $\rho$ is a potential increment and $D$ is a diffusion coefficient, with $0<\rho<D, 0<D<1$. After some setting time, the farther a node $n$ is from destination $d$, the higher $V^{d}(n, t)$ is. Routing is achieved using (2) :

$$
\operatorname{nexthop~}^{d}(n)=\left\{k \mid k \in n b r(n) \wedge F_{k}^{d}(n)>\beta\right\}
$$

with

$$
F_{k}^{d}(n)=V^{d}(n)-V^{d}(k)
$$

$F_{k}^{d}(n)$ is the force that affects a message send to $d$ from node $n$ toward node $k . \quad \beta$ is a threshold set to 0 in our experiment. We implemented PEAR MCS (Multiple Candidate Selection) [3], in which the packet can be relayed to multiple node.

This routing algorithm only requires local information to work (the potential of the direct neighbors of a node), which limits the amount of signaling to do. The biggest part of this signaling is the diffusion of the potential values between neighbors. But for networks with very few data packets, the signaling can be more resource-consuming than actual data transmission.

In order to compare our results with a well-known algorithm, we also implemented a slightly optimized epidemic routing. In this model, packets are immediately stored and forwarded to every neighbor using a copy management algorithm. If a node receives a "discovery \& fetch" message, it forwards to the sender of this beacon every message the latter has not already received. Only the mobile node and the base station were sending these messages.

\section{IMPROVING POTENTIAL DIFFUSION ALGORITHM}

\section{A. Naive potential diffusion}

In [3], it is suggested that every node periodically (e.g. every $10 \mathrm{~s}$ ) sends its own PEAR potential information to its neighbors to let them refresh their own potential table (which we call the "naive" potential dissemination). A tradeoff must be done between a short interval between potential diffusion messages for network reactivity and a long period for low network consumption. In our simulations, these message were send every 600s to match our time scale without impairing too much the reactivity.

\section{B. Proposed improvements}

In order to reduce the number of signaling messages send by the network level, we propose an new potential diffusion algorithm for PEAR, which we call PEAR OPD (Optimized Potential Diffusion). Because the potential diffusion spreads information about topology over the network, we limit the diffusion of this information to the moment when the network's topology is actually changing, i.e. when a mobile node encounters another node.

Between two potential diffusion messages, the node makes the assumption that the neighboring nodes updates their potential as if they were alone : it estimate their potential with the formula $V^{d}(n, t+1)=V^{d}(n, t)+\rho$ then updates its own potential according to (1), with the estimated potentials.

When a node sends a potential diffusion message, it starts a chain of diffusion : its direct neighbor will in response also send their potential diffusion message, which will trigger a potential diffusion by the next neighbor, and so on. Because these message are not stored, the chains only propagate within one sub-area. 
In order to avoid loops in these chains, we also implemented a "chain copy management" based on our copy management algorithm : upon reception of a diffusion message, the duet \{first sender in the chain, chain number\} is recorded, and any following potential message matching these values will be discarded. Thus, the relevant potential information is disseminated over the whole sub-area, and loops are avoided. The algorithm was implemented according to the following :

(1) Upon reception of a diffusion message, the receiving node checks if the duet \{first sender, chain number $\}$ is known. If not, it records it, otherwise it drops the message.

(2) It performs the update of its own potential table.

(3) It replaces in the message the previous potential information with data relative to itself, leaving the initial sender ID and chain number.

(4) It send this message to every neighbor.

This mechanism provides better results than a TTL limitation because short loop avoidance requires low TTL initial value, whereas high networks depth requires high TTL initial value to ensure data dissemination over the whole subarea. Furthermore, this initial value should be re-optimized for every new topology.

To match the fact that potential information should only be send when topology is changing, only the mobile node sends periodically these "first chain message". These messages were send every $20 \mathrm{~s}$ in our simulations to ensure at least one connection each time a mobile node passes by a static node.

\section{EVALUATION OF THE IMPROVEMENTS}

\section{A. Conditions}

In our scenario, every field is a $500 \mathrm{~m}$ wide square (as drawn in Fig. 1) and the vehicle travels along a path at $6 \mathrm{~m} / \mathrm{s}$. It takes one day to perform one trip, then it repeats the trip. Each field is equipped with 20 nodes, placed on a grid, $100 \mathrm{~m}$ apart, e.g. near the tractor's tracks. In our 7 days simulations, the sensor nodes send data during days 3 and 4. During the first 2 days only the vehicle is moving in order to set up the potential field. After a random initialization time (uniform over period second), each node sends a data packet of size bytes every period second (typical values : size $=100 \mathrm{~B}$, period $=$ $3600 \mathrm{~s})$.

The nodes have a range of $120 \mathrm{~m}$, and a maximum datarate of $20 \mathrm{kB} / \mathrm{s}$. The mobile node spends about $30 \mathrm{~s}$ withing range of the static node it encounters (except for the base station). The MAC used here is a CSMA. Each node was also provided with a buffer, large enough to store 30000 messages. This assumption is realistic given the low cost of flash memory, a few gigabytes are now affordable for such devices.

\section{B. Simulation results}

Our network was simulated using the Omnet++ simulator [5] [6], with the tools from MiXiM [7] and INET [8] projects.

\section{1) Trafic-free network measurement}

We first measured the network consumption for both naive and optimized potential diffusion in a network without any application layer data. In this case, only the trafic-independent signaling is send.

The results, reported in table I, show us that for a naive potential diffusion period of 600s, naive algorithm sends about 27 time more packets and bytes than the optimized one. We reach the same consumption for both naive and optimized with a $16200 \mathrm{~s}(4,5 \mathrm{~h})$ potential diffusion period for naive. This would however lead to a low network reactivity, so a $600 \mathrm{~s}$ period was used in the other experiments.

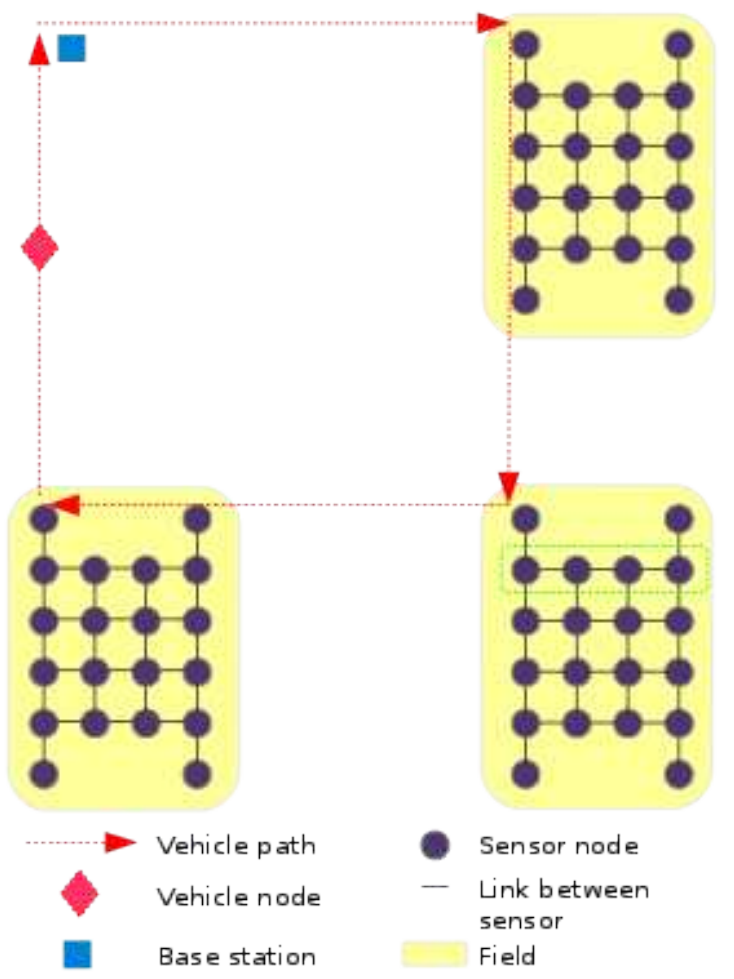

Fig. 1. Topology of the network (not to scale). The vehicle spend one third of every day ( $8 \mathrm{~h})$ stopped within range of the base station

TABLE I. BASE NETWORK CONSUMPTION

\begin{tabular}{|c|c|c|}
\hline $\begin{array}{c}\text { PEAR diffusion } \\
\text { algorithm }\end{array}$ & Packets send & Bytes send \\
\hline OPD & 2191 & 28483 \\
\hline Naive $(600 \mathrm{~s})$ & 59143 & 758606 \\
\hline Naive $(16200 \mathrm{~s})$ & 2183 & 22553 \\
\hline
\end{tabular}




\section{2) Successful delivery rate}

In the next experiment, we ran the network under different loads by changing the period between 2 application data packets (with a size of data packets of $100 \mathrm{~B}$ ), or by changing the size of data packets (with a period of $3600 \mathrm{~s}$ ). The delivery success rate for this experiment (Fig. 2 and Fig. 3) shows that for both naive and OPD, we reached almost the same successful transmission rate than epidemic. For the typical values used in this study (100 Bytes every 3600 s), we have respectively $99.1 \%, 95.3 \%$ and $98.2 \%$ successful transmission rate for PEAR OPD, PEAR with naive potential diffusion and Epidemic. It complies with the previous studies made on PEAR algorithm, and proves that the parameters used in our simulations gives our system the performances requiered for our application.

The successful transmission rate is low for high values of the size of data packets because the maximum datarate of our sensor nodes was quite low $(20 \mathrm{kB} / \mathrm{s})$ compared to the size of a packet. For $10 \mathrm{kB}$ packets, one can at most transfer 60 packets during the $30 \mathrm{~s}$ contact time with the mobile node, whereas 480 messages are generated within a 20 nodes field every day and should be transmitted during these $30 \mathrm{~s}$. Likewise, this rate is low for short period values because of the limited speed of the link between two nodes.

\section{3) Network efficiency}

In Fig. 4 and Fig. 5 is represented what we call the "packet efficiency" (defined in (5) ) of the network and in Fig. 6 and Fig. 7 is represented the "byte efficiency" (defined in (6) ) of the network. In these formula, the "total amounts" are computed by counting the packets (or bytes) send by sensor nodes for the whole network.

$$
\begin{gathered}
\text { packet efficiency }=\frac{\text { amount of delivered packets }}{\text { total amount of send packets }} \\
\text { byte efficiency }=\frac{\text { amount of delivered bytes }}{\text { total amount of send bytes }}
\end{gathered}
$$

Figures 4 to 7 show us that the optimized potential diffusion algorithm improves both packet and byte efficiency compared to the naive diffusion algorithm. It also shows us that, under the experiment conditions (few small data packet transmitted), the naive approach performs worst than pure epidemic, whereas the optimized approach displays better performances than epidemic in terms of consumed networks resources, provided that the network is loaded enough.

According to the previous assumption (cf. II.B), we can say that the energy consumption of the whole network will always be lower for PEAR OPD compared to PEAR with naive potential diffusion.

\section{4) Latency}

Figure 8 represents the mean delay in the network. We can see that one of the side effect of OPD, apart from reducing network consumption, is to bring overall latency to a value close to the shortest possible (given by Epidemic routing).

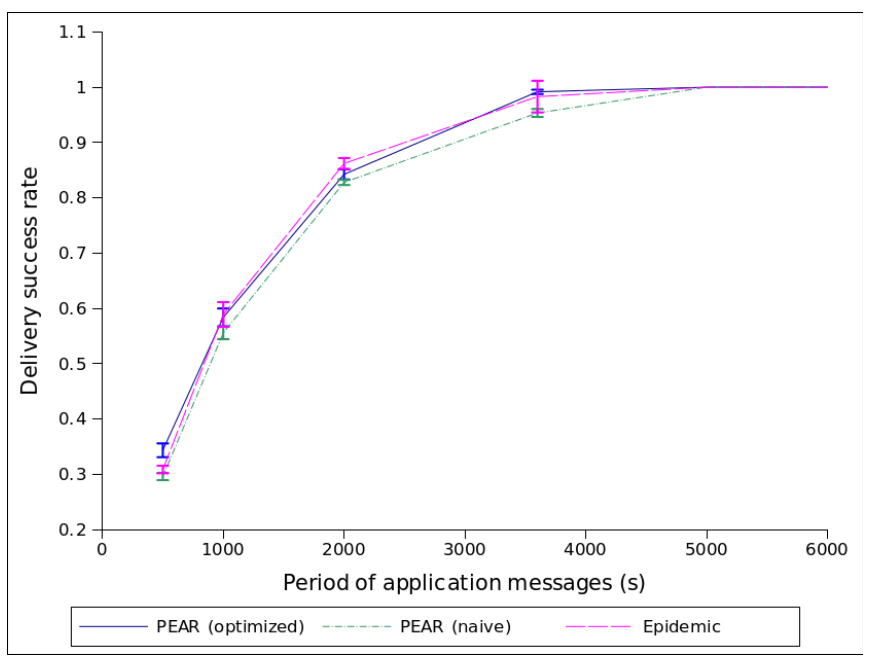

Fig. 2. Delivery success rate vs period of application messages

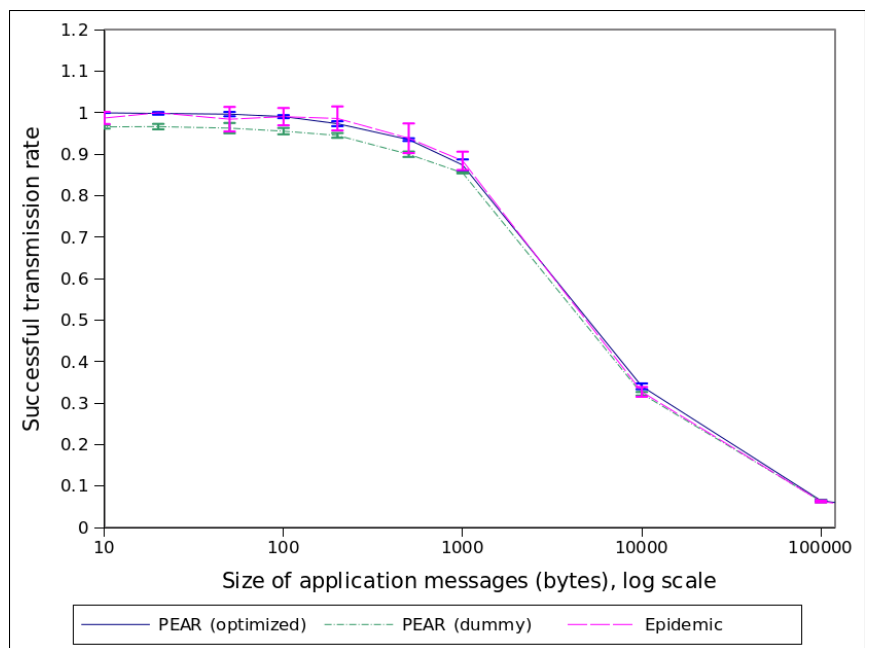

Fig. 3. Delivery success rate vs size of data message

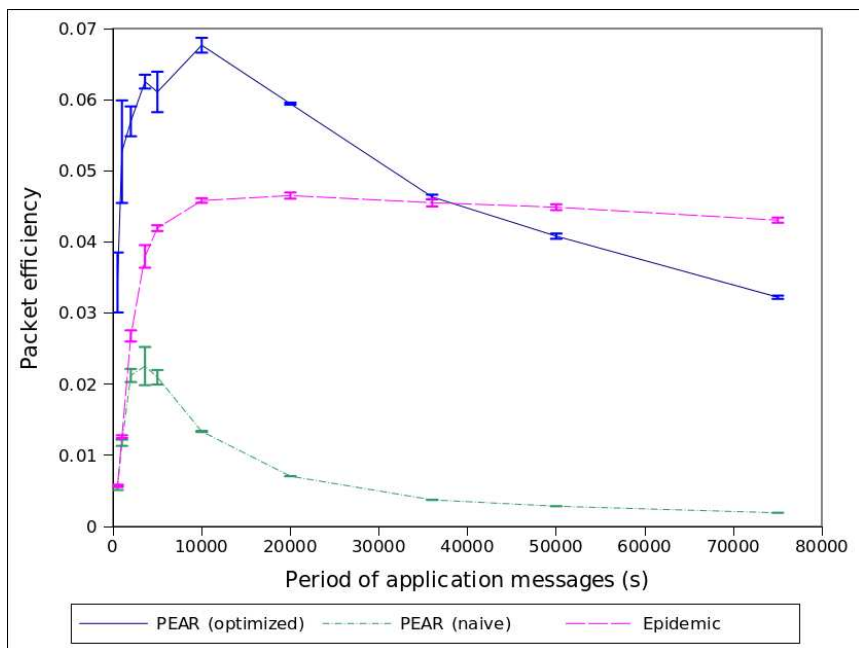

Fig. 4. Packet efficiency vs period of application messages 


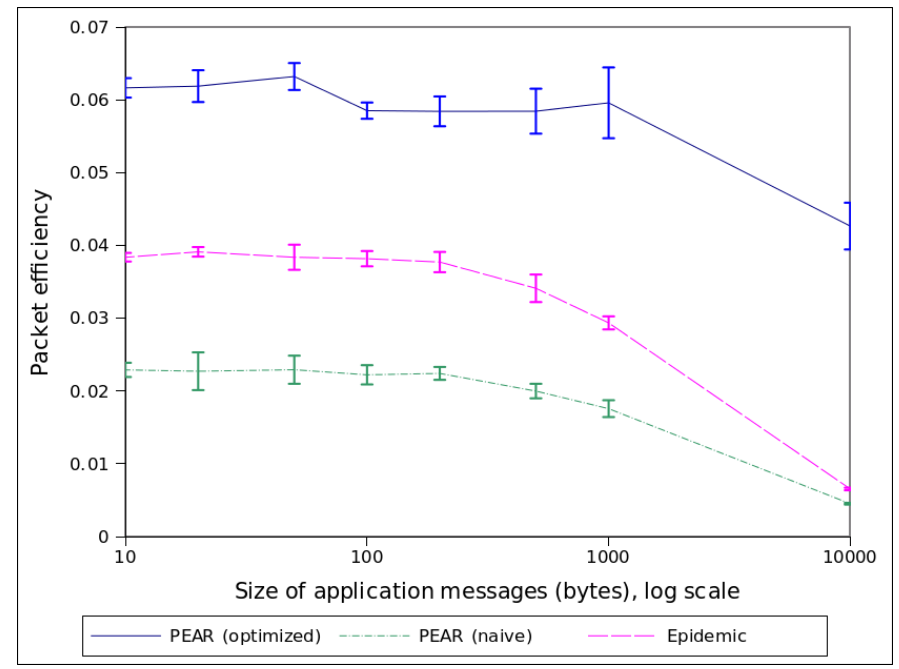

Fig. 5. Packet efficiency vs size of data message

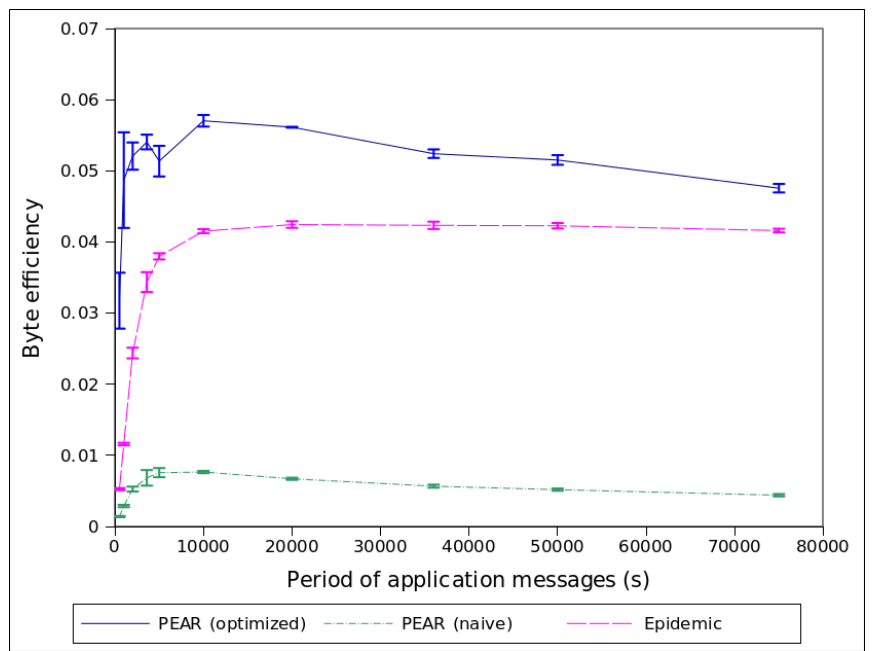

Fig. 6. Byte efficiency vs period of application message

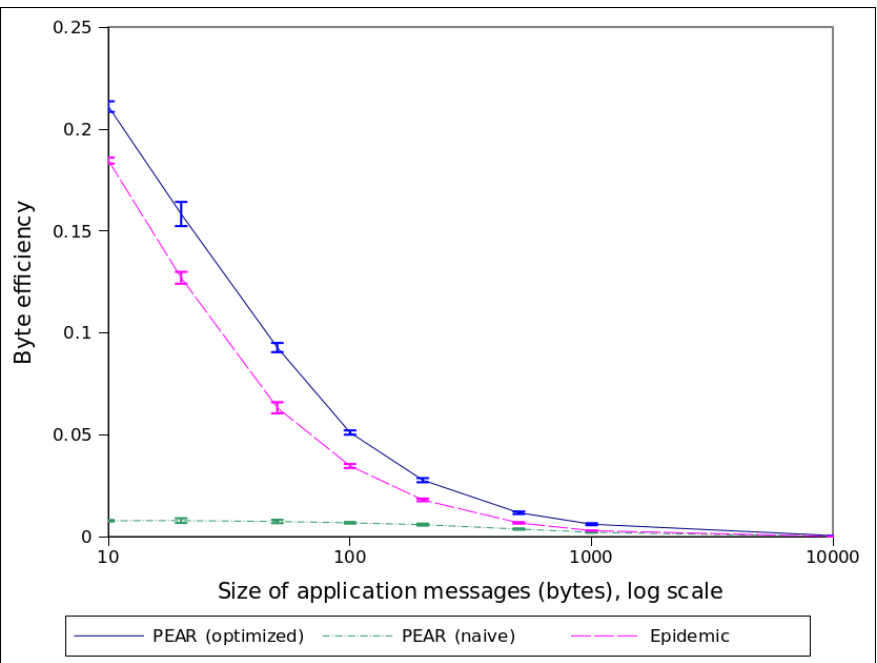

Fig. 7. Byte efficiency vs size of data message

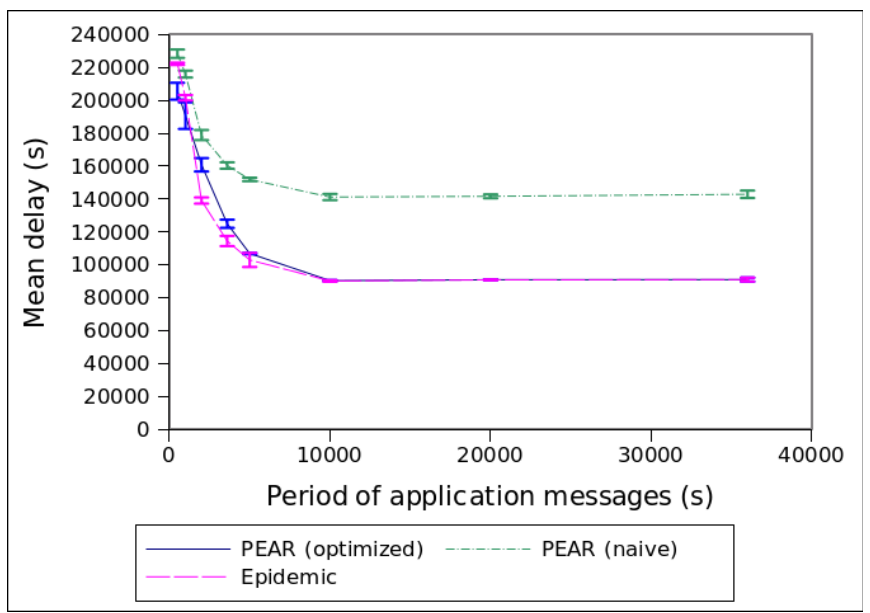

Fig. 8. Mean delay vs period of application message

\section{5) Message repartition}

Figure 9 demonstrates that signaling messages represents a large amount of the total number of messages send for the naive potential diffusion, whereas they are negligible for PEAR OPD.

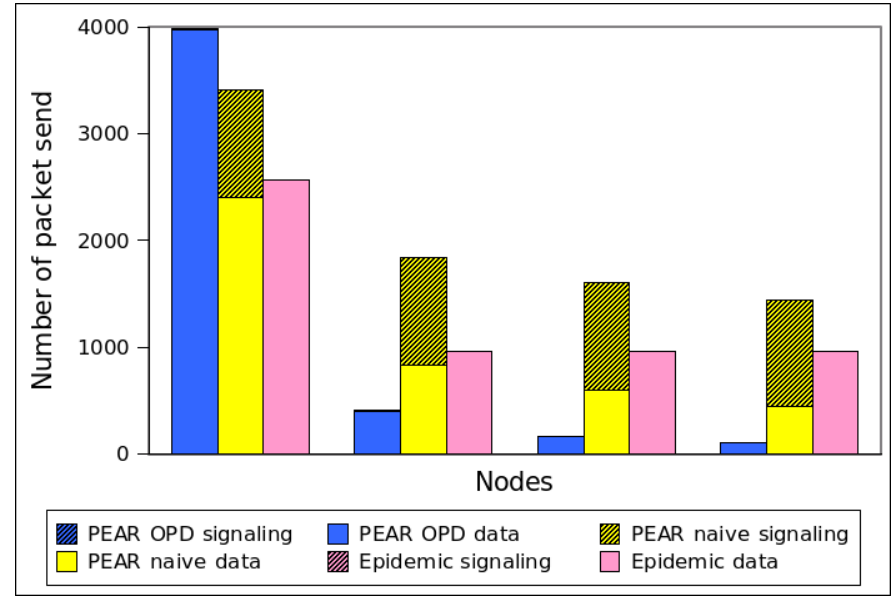

Fig. 9. Message repartition for different nodes and algorithm. these four nodes correspond to the nodes within the green dotted line in Fig. 1, the left node on the above graph being the closest to the path of the mobile node in Fig. 1.

\section{DISCUSSION AND RELATED WORK}

Our simulations have demonstrated that the PEAR routing algorithm with naive potential diffusion performs, in networks with very low loads, worst than a simple epidemic routing. We proposed a new way of transmitting this information, which we called PEAR OPD, and demonstrated that its transmission rate and latency are very close to Epidemic performances under our typical conditions. Its energy efficiency has also been demonstrated, PEAR OPD always brings an improvement compared to PEAR naive in terms of amount of packets send or byte send. More specifically, the packet efficiency is 
multiplied by 2.77 compared to PEAR naive, and the byte efficiency is multiplied by 7.9, for our typical traffic values.

We recognize that these values are heavily dependent on the load of the network but we can anyway draw the following conclusion : the usefulness of PEAR OPD against Epidemic increases when :

- The frequency of data message increases.

- $\quad$ The size of data message increases.

We can also draw some conclusion on the required MAC protocol that should be used in this kind of networks. Because of the specific connectivity (only $30 \mathrm{~s}$ every day), the ideal MAC for this kind of network should prioritize burst transmissions between mobile and static node to maximize successful transmission rate, and remain energy efficient the rest of the time.

In the experiment [4], the considered scenario was similar to ours, but with some differences. First, they had only 5 sensors emitting data every 30s, whereas each sensor node of the 60 sensors in our simulation was generating one packet every 3600s. We think that a 30s time-granularity is not required for agricultural monitoring, and this frequency could be reduced using pre-processing, data emission only on changes or data aggregation and compression at the sender's level. Second, we also had only one mobile node instead of 7, and it was traveling on a fixed path. Our mobility scheme corresponds to a vehicle traveling along the fields on a country road, not necessarily working within the field. Finally, the throughput limitation of one packet per hour for each node in our case is due to the fact that the maximum datarate is $20 \mathrm{kB} / \mathrm{s}$ and the contact time is limited (1 slot of about 30 s per day). In [4], WiFi link were used, which provides better throughput, and the mobiles nodes were probably in contact with static nodes more often.

\section{CONCLUSION}

In this paper a new signaling data dissemination algorithm is proposed to reduce the energy consumption induced by the signaling of PEAR routing algorithm in a low loaded DTN Wireless sensor network. It was proved, with simulations, that this algorithm, PEAR OPD, improves in every case the network efficiency and reduces the network energy consumption compared to a naive diffusion, allowing the use of less energy-greedy nodes. It was also demonstrated that PEAR OPD improves significantly the delay in the network compared to PEAR with naive diffusion. PEAR OPD has the same successful transfer rate and latency than Epidemic, but has a better efficiency and lowest consumption under our typical conditions. This algorithm brings a new energy efficient routing for large scattered networks generating data at a low rate.

\section{REFERENCES}

[1] Alex Pentland, Richard Fletcher, and Amir Hasson, "DakNet: Rethinking Connectivity in Developing Nations,” IEEE Computer Society, pp. 4-9, Jan-2004.

[2] P. Juang, H. Oki, Y. Wang, M. Martonosi, L. S. Peh, and D. Rubenstein, "Energy-efficient computing for wildlife tracking: design tradeoffs and early experiences with ZebraNet,” SIGOPS Oper. Syst. Rev., vol. 36, no. 5, pp. 96-107, Oct. 2002.

[3] H. Ochiai and H. Esaki, "Mobility entropy and message routing in community-structured delay tolerant networks," in Proceedings of the 4th Asian Conference on Internet Engineering, New York, NY, USA, 2008, pp. 93-102.

[4] H. Ochiai, H. Ishizuka, Y. Kawakami, and H. Esaki, “A DTN-based sensor data gathering for agricultural applications,” Sensors Journal, IEEE, vol. 11, no. 11, pp. 2861-2868, 2011.

[5] “OMNeT++." [Online]. Available: http://www.omnetpp.org/. [Accessed: 15-Mar-2013].

[6] E. Egea-Lopez, J. Vales-Alonso, A. S. Martinez-Sala, P. Pavon-Marino, and J. García-Haro, "Simulation tools for wireless sensor networks," in Proceedings of the International Symposium on Performance Evaluation of Computer and Telecommunication Systems (SPECTS'05), 2005.

[7] "MiXiM." [Online]. Available: http://sourceforge.net/apps/trac/mixim/. [Accessed: 15Mar-2013].

[8] “INET." [Online]. Available: http://inet.omnetpp.org/. [Accessed: 17-Mar-2013]. 